\title{
Motor Control Abnormalities in Parkinson's Disease
}

\author{
Pietro Mazzoni, Britne Shabbott, and Juan Camilo Cortés \\ Motor Performance Laboratory, The Neurological Institute, Columbia University, New York, \\ New York 10032 \\ Correspondence: pm125@columbia.edu
}

\begin{abstract}
The primary manifestations of Parkinson's disease are abnormalities of movement, including movement slowness, difficulties with gait and balance, and tremor. We know a considerable amount about the abnormalities of neuronal and muscle activity that correlate with these symptoms. Motor symptoms can also be described in terms of motor control, a level of description that explains how movement variables, such as a limb's position and speed, are controlled and coordinated. Understanding motor symptoms as motor control abnormalities means to identify how the disease disrupts normal control processes. In the case of Parkinson's disease, movement slowness, for example, would be explained by a disruption of the control processes that determine normal movement speed. Two long-term benefits of understanding the motor control basis of motor symptoms include the future design of neural prostheses to replace the function of damaged basal ganglia circuits, and the rational design of rehabilitation strategies. This type of understanding, however, remains limited, partly because of limitations in our knowledge of normal motor control. In this article, we review the concept of motor control and describe a few motor symptoms that illustrate the challenges in understanding such symptoms as motor control abnormalities.
\end{abstract}

$T_{\text {dis }}^{\text {h }}$ he effects of Parkinson's disease (PD) can be described at different levels. Within the brain, the major pathological change is progressive degeneration of neurons in the pars compacta of the substantia nigra, one of the nuclei that constitute the basal ganglia (BG). These neurons normally transmit dopamine to another BG nucleus, the striatum, but their degeneration leads to dysfunction of these neuronal circuits that include the BG and motor cortical areas. At the level of an individual's behavior, these changes result in movement abnormalities, which are the major manifestations of the disease. These difficulties, in turn, cause major disruptions that range from an individual's quality of life to society-wide economics. Our goal in this article is to describe motor symptoms of PD at the level of motor control. We briefly review what is meant by "motor control" and describe the process of understanding a symptom as a motor control abnormality. We then focus on selected symptoms that, among the many and varied motor symptoms of PD, have been most studied from a motor control perspective.

Editor: Serge Przedborski

Additional Perspectives on Parkinson's Disease available at www.perspectivesinmedicine.org.

Copyright (C) 2012 Cold Spring Harbor Laboratory Press; all rights reserved; doi: 10.1101/cshperspect.a009282

Cite this article as Cold Spring Harb Perspect Med 2012;2:a009282 
P. Mazzoni et al.

\section{MOTOR SYMPTOMS OF PARKINSON'S DISEASE}

PD can cause a variety of motor symptoms and signs, several of which are listed in Table 1. Note that we will interchangeably use the terms "symptom" (a disease manifestation experienced by the patient) and "sign" (a disease manifestation detectable by a clinician) in this article because the distinction between these terms

Table 1. Selected motor symptoms and signs of Parkinson's disease

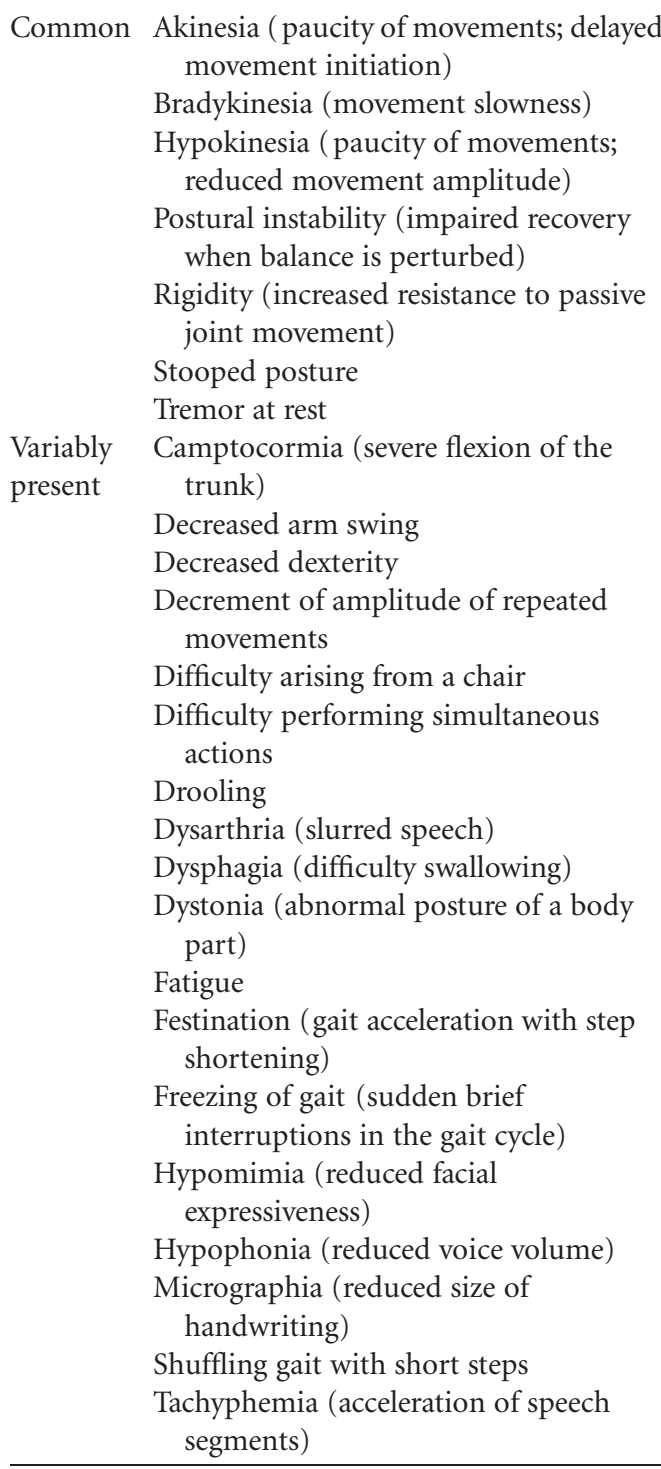

does not affect the present discussion. Several symptoms (Table 1, "Common") are very common and appear in most patients at some point in the course of the illness. Bradykinesia, akinesia, and hypokinesia are nearly universal symptoms, in that they eventually appear in nearly every patient. They are typically present early in the course of the disease. Although the usage of these terms varies, "bradykinesia," "akinesia," and "hypokinesia" generally refer to a paucity and generalized slowing of movements in the absence of weakness (Hallett 1990; Fahn 2003). Movements take more time, and there is reduced frequency of spontaneous movements, such as blinking, smiling, and grimacing, which gives the face a mask-like, expressionless appearance. In this article, we use "bradykinesia" in reference to movement slowing; "hypokinesia" for reduction of movement amplitude (and/or force); and "akinesia" for the two phenomena of paucity of movements and delayed movement initiation. "Postural instability" refers to impaired reaction when balance is perturbed. For example, tripping on an uneven sidewalk may lead to a fall because the patient's response is inadequate for recovery of balance. "Rigidity" refers to increased muscular tone. There is more resistance than normal when the limb is passively moved (e.g., by a clinician). Rigidity is usually experienced as a sense of feeling stiff and uncomfortable. "Stooped posture" is an abnormal posture marked by shoulder dropping and head bowing. "Rest tremor" is a repetitive back-and-forth movement of any limb, or the jaw, head, or trunk, which occurs when that part of the body is not actively moving. Common types of tremor include pronation-supination of the forearm and flexion-extension of the fingers. Some patients with PD never develop tremor.

The remaining symptoms (Table 1, "Variably present") are of variable occurrence. An interesting aspect of these symptoms is that many appear related, at least superficially, to the "Common" symptoms listed in Table 1. Hypomimia (masking of facial expression), for example, has already been mentioned as a manifestation of akinesia-hypokinesia, and micrographia (decreased size of handwriting) is likely part of the same symptom complex. Drooling is 
likely not due to excessive saliva production, but rather to a combination of reduced swallowing frequency and a tendency for the mouth to stay partly open, which are manifestations of akinesia-hypokinesia-bradykinesia.

It is not entirely clear, however, to what extent PD symptoms are related to one another. Consider the case of movement slowing of individual movements (bradykinesia) and slowing of sequential and simultaneous movements. When asked to make a series of movements, PD patients perform each movement abnormally slowly, but also wait longer than normal between each movement (Benecke et al. 1987). When asked to perform two movements simultaneously, the amount of slowing is greater than would be expected if one simply added together the slowing of each individual movement. It is difficult to discern whether this effect truly represents a separate difficulty from bradykinesia. A normal action, such as getting dressed, includes multiple simultaneous and sequential tasks. If these are executed less simultaneously and with longer intervals between each movement, the total movement time can easily become considerably long (Schwab et al. 1954).

A difficulty in interpreting the slowing of sequential and simultaneous movements for $\mathrm{PD}$ patients is that comparison with normal performance is not trivial. It is possible that controlling the sequential and simultaneous performance of movements that are individually slow takes extra time because they are controlled differently from normal movements. Thus the extra time needed to execute sequential or simultaneous movements could be due not to $\mathrm{PD}$, but a general difficulty in sequential or simultaneous execution of slow movements. To distinguish between these possibilities, one would, ideally, slow the individual movements of healthy subjects artificially to match the slower speed of patients' individual movements, and then record how fast these subjects can execute those slow movements sequentially or simultaneously. If they did not show additional slowing, then one could conclude that PD patients' difficulty with sequential and simultaneous movements is a separate motor abnormality from bradykinesia.
One consideration for understanding how symptoms might be related is whether symptoms are correlated to each other in their occurrence and severity. The idea is that if two symptoms result from the disruption of a common normal mechanism, then they should co-occur. This approach is helpful for symptoms that affect the same body region. The lack of correlation between severity of bradykinesia and rigidity, for example, suggests that these symptoms constitute separate motor abnormalities, and specifically, that bradykinesia is not simply a consequence of rigidity (Marsden 1989). This reasoning, however, is less useful when considering symptoms that affect different body regions. Indeed, based on correlation, micrographia and postural instability should be considered unrelated, because PD often begins by affecting an upper limb first, and only later affects gait and balance. However, PD symptoms often have a particular topography that depends on the stage of the disease, that is, they affect a given body region first and then spread to adjacent regions. It is therefore possible for micrographia and postural instability not to coexist, simply because the arm has been affected but gait has not. And yet, it is quite plausible that the two symptoms might both reflect hypokinesia. Reduced movement amplitude can cause handwriting to be smaller. Reduced movement amplitude can also cause a step to be smaller, so that if a patient trips over an obstacle, she might not recover because she does not take a large enough step to avoid falling.

A more reliable approach to understanding how motor symptoms relate to one another is to determine, if possible, how they relate to normal motor control. For example, decreased arm swing could result from rigidity, because of increased resistance to passive movements. However, the arm swing is not only a passive movement, but also has an active component from coordinated muscle contractions (Elftman 1939). Akinesia/hypokinesia, therefore, could also contribute to arm swing reduction. These potential relationships beg the questions: How is arm swing amplitude normally controlled? What is the role of normal tone in enabling normal-amplitude movements? 
P. Mazzoni et al.

Understanding relationships among PD symptoms is only one reason to try to investigate the nature of these symptoms as motor control abnormalities. More generally, motor symptoms represent functional impairments and are mixed with compensatory strategies to cope with them. Treatment like medications, physical therapy, and neurosurgical interventions are designed to restore normal motor abilities. Understanding the nature of motor symptoms as motor control abnormalities can help to establish the functional effects of the disease on various motor functions and can help to optimize therapeutic strategies. In addition, an important direction for the treatment of brain disorders is the development of neural prostheses, that is, artificial circuits that can replace the function of damaged brain regions (Brunner et al. 2011). Existing ones, such as artificial cochleas (Liu and Delbruck 2010), were designed based on the normal function of biological cochleas. Designing artificial basal ganglia will require understanding how these structures contribute to normal motor control and how their dysfunction causes specific motor symptoms.

\section{MOTOR CONTROL}

To gain an understanding for how motor control is affected in Parkinson's disease, it is worth reviewing what is meant by "motor control." Many of the following concepts were explicitly introduced by Nikolai Bernstein, in a landmark book that was translated into English in 1967 (Bernstein 1967). Modern accounts of motor control can be found, for example, in Winter (2009) and Shadmehr and Wise (2005). In general, movements have features that are tightly controlled and others that require less precise control. For instance, when you are sitting at a banquet and reach for a glass of wine, your hand travels along a path through three-dimensional space. The wrist and elbow also travel through space, and yet their position is less important, because it is the hand that must reach the glass. Conversely, when we elbow our spouse at the table to alert them that someone important just walked into the banquet hall, the hand moves in space, but its position matters less than that of the elbow, because it is the elbow that must hit our dinner companion's ribs. It is thus useful to describe what feature is controlled: hand position when we reach, and elbow position when we nudge. The motor system faces the task of controlling various aspects of movement, as dictated by specific task demands. Some movement features may be tightly controlled, such as the final position of the effector (hand or elbow), whereas others may be controlled more loosely. In other tasks, multiple features may need to be tightly controlled. When one is kicking a soccer ball, the foot's speed, the point of contact of the foot on the ball, and the angle of the hip are all crucial to the task's goal. The motor system also controls movement features outside the body, such as the trajectory and speed of the head of a hammer when hitting a nail.

Controlling different aspects of movement is not trivial, given that the nervous system cannot directly influence the position of hand, elbow, foot, or hammer head. It can only cause muscle contractions, which result in forces that cause joint rotation. Achieving a desired movement requires devising the desired path of the relevant moving body part (such as hand trajectory when reaching for an object); translating this movement goal into the appropriate set of muscle activations; accounting for how other body parts (such as the elbow) will move along; and monitoring the movement as it unfolds in case corrections are needed. The nervous system must also account for external factors that influence movement goals (such as the speed of an oncoming soccer ball). These are all components of motor control.

To understand motor control abnormalities in $\mathrm{PD}$, one would, ideally, start from a detailed understanding of normal motor control and then infer what changes to control strategies are necessary to explain PD motor symptoms. In practice, this approach is difficult because our understanding of normal motor control is limited. In particular, we have limited understanding of the normal motor functions that are particularly affected by PD. For bradykinesia, for example, we would need to understand how movement speed is normally selected; for rigidity, what control principles dictate normal muscle tone. 


\section{MOTOR SYSTEM: LEVELS OF DESCRIPTION}

Our concern is to address the relationship between motor symptoms and motor control. This is a distinct endeavor from reviewing the neurophysiology of motor symptoms, although the two are closely related. The distinction is perhaps best described in the language of David Marr's levels of description of an information processing system (Marr 1982). The motor system can be considered such a system, in the sense that it receives information (e.g., location of an object to be reached, current position of all body parts, behavioral goals) and produces outputs (muscle activations). Therefore, like all information processing systems, the motor system can be described at three levels: computational, algorithmic, and physical.

The computational level is a description of what the system does and why. In the case of reaching movements, the motor system enables one to accomplish a behavioral goal, such as procuring a glass of water. The hand's trajectory has certain features. It is mostly straight, reaches peak velocity near the middle of the movement, and has a particular duration. There are likely important reasons for moving in this manner. A straight trajectory takes less time and effort than a curved trajectory. Thus, trajectories may be straight in order to minimize time or energy. Minimizing a quantity is an example of a computational principle, which provides insight as to why the computation is performed in a certain way. Theories that attempt to explain the features of real movements are computational theories, and developing and testing computational theories is the concern of the discipline of motor control.

The other two levels of description are the algorithmic and implementation levels. Whereas the computational level describes what the motor system does and why, algorithms describe how the goals are accomplished. For a reaching movement, the motor system might first represent the desired trajectory in space and then activate muscles as needed in order to keep the hand on course along the desired path. The implementation level describes the operation of the physical structures (the hard- ware) that actually perform the computation. The motor system's hardware mainly consists of neurons and muscles, which communicate via electrical impulses and chemical substances and are connected together as circuits.

Bradykinesia can illustrate the distinction between the computational level and algorithm/ implementation levels. Slowing of movement in PD is associated with abnormal balance of activity among multiple parallel pathways within the BG (Alexander et al. 1986; Albin et al. 1989; Obeso et al. 2008). This abnormal balance is a consequence of a reduction in dopamine signals sent from the substantia nigra to the striatum, and the net result is excessive activity in BG's output activity, which reduces activation of the motor cortex for a given movement. Such a description is at the hardware level. It tells us how the circuitry behaves differently from normal. A description at the algorithmic level is that the BG in PD fails to sufficiently energize the motor cortex to produce a movement of normal speed (Hallett and Khoshbin 1980; Hallett 2003). What is needed at the computational level of description is a theory of how movement speed is normally determined. What principles (maximization of movement accuracy, minimization of energy) influence movement speed determination, and how does dopamine loss in PD disrupt these computations?

The different levels are, of course, related, and a clean distinction among them is not always possible or appropriate. But brain circuits and their activity must implement algorithms that in the end must subserve computations. A full understanding of motor dysfunction in PD will be obtained when we can describe the normal computations and algorithms that are implemented by the motor system and how the physical changes caused by PD disrupt these algorithms and, in turn, the computations that normally govern movement.

Our understanding of motor symptoms of PD has made phenomenal advances in terms of kinematics (how are movements different in PD?) and neurophysiology (Hallett 2003), which are largely concerned with the second and third levels of description, respectively: algorithm and 
P. Mazzoni et al.

physical realization. The major approach has been to link the nature of dysfunction in neuronal circuits and activity (i.e., the disruption of the hardware) to motor abnormalities or symptoms. A comprehensive description of $\mathrm{PD}$ motor symptoms at the computational lev$\mathrm{el}$, on the other hand, remains elusive. A template for this type of understanding exists for a motor symptom that is not part of PD, namely, cerebellar ataxia (Box 1). Here we describe how two major symptoms, rigidity and the complex of bradykinesia-hypokinesia-akinesia, may be understood as abnormalities of normal motor control.

\section{RIGIDITY AND MOTOR CONTROL}

When a joint is moved passively (i.e., by the examiner, without any effort by the patient to help or resist the motion), there is a normal amount of "tone" (resistance to passive movement). "Rigidity" refers to increased tone, or increased resistance to passive movement. To understand rigidity as a motor control abnormality, we must ask what is the role of muscle tone in motor control. The full answer to this question is not yet clear, but normal tone is likely part of a general mechanism for maintaining stability, that is, resistance to perturbations. When a limb is at rest and relaxed, the

\section{BOX 1. FROM MOTOR SYMPTOM TO MOTOR CONTROL}

It has long been a tradition in neurology to infer normal nervous system function from analysis of neurological symptoms. It may seem almost obvious that a neurological abnormality might directly reflect the loss of a corresponding normal function. Weakness after a corticospinal tract lesion might lead to the conclusion that the motor cortex controls muscle power. In reality, translating symptom into normal function is a complex process, full of logical traps. Although muscle power does require proper activation patterns in motor cortex and motor cortex stimulation causes muscle contraction (Ferrier 1876), motor cortical activity might encode other aspects of movement, such as speed, that happen to correlate with muscle power. Indeed, activity in motor cortex is correlated with so many aspects of movement (Thach 1978; Scott 2003) that it remains unclear, more than 130 years after Ferrier's stimulation experiments, what role the motor cortex plays in motor control (Scott 2008).

What does it mean to understand a symptom in terms of motor control? It may be helpful to describe an example in which this type of understanding has arguably been achieved. Consider the symptom of "cerebellar ataxia." This term refers to the fact that patients with cerebellar disease make "irregular" movements. For example, movements of the finger from the patient's nose to the examiner's fingers weave along an irregular path to the target. Furthermore, when a movement is repeated, the path traced weaves along a slightly different trajectory, compared with the previous one. How are we to interpret ataxia in terms of normal cerebellar function? By noticing that the finger path is not straight, we are implying that finger paths in goal-directed movements are normally straight, leading one to consider that the cerebellum's normal function is to control the straightness of the trajectory. To a large extent, however, this is simply a restatement of the neurological symptom in the negative. A more mechanistic hypothesis is needed.

Briefly, key insights arose from the ideas that muscle contractions and relaxations in cerebellar patients seem delayed (Holmes 2007) and that these patients have difficulty managing interaction torques (the reactive forces between limb segments, such as between the forearm and upper arm) (Bastian et al. 1996). These explanations were insightful, but what was missing was a theory of how and why delays and interaction torques mattered in normal motor control.

A breakthrough came from the field of computational motor control, which is concerned with identifying principles and mechanisms that operate in normal motor control. The novel hypothesis was that the motor system handles delays, interaction torques, and other factors by computing a "forward model" (Kawato et al. 1987; Wolpert et al. 1995; Miall and Wolpert 1996). The joint forces needed early in the movement, for example, differ from those needed in the middle, where the arm is moving faster. This higher speed causes mechanical forces between the upper arm and forearm, and the motor command must be adjusted to account for these forces. But the adjusted motor command 
will only arrive at the muscle after it has traveled through central and peripheral nerves $(\sim 100-$ $130 \mathrm{msec}$ ). A "forward model" refers to an internal computation through which the motor system accounts for these changing conditions and delays. It monitors the state of the arm (its position and velocity) and sends adjustments to the motor command that take into account transmission delays. Crucially, the computation involves a model of the arm that allows predicting, based on the most recently sensed arm position and velocity, where the arm will be by the time the motor command reaches the muscles. By maintaining a model of the arm that takes into account neural transmission delays, the motor system can exert predictive control.

It soon became apparent that many motor abnormalities due to cerebellar dysfunction could be explained as a disruption in predictive control. It was thus hypothesized that in normal motor control, the forward model is computed by the cerebellum (Miall et al. 1993; Bastian 2006). The idea is that a normal reaching movement requires motor commands that take into account the biomechanical properties of the arm, the arm's changing state during the movement, and delays in signal transmission. The cerebellum normally achieves predictive control by taking all these factors into account, making it possible for the hand to follow a straight path. Disrupting cerebellar function then leads to irregular hand paths because motor commands are no longer predictive and are not appropriate for the current state of the arm. The path irregularity varies from one movement to the next because inappropriate adjustments to the motor command may vary in their nature and their effect depending on exactly when they are released, and because their effects accumulate throughout the movement.

What is successful about our current understanding of cerebellar ataxia is that a motor control principle was identified (the need for predictive control through a forward model) that can account for important features of normal movements, and whose disruption can explain motor symptoms of cerebellar dysfunction. Predictive control through a forward model is a crucial component of computational models of normal motor control. As a principle, it may have historically been suggested by the symptoms of cerebellar dysfunction, but those symptoms can now be explained as disruptions of the principle of predictive motor control.

muscles are not active. If an external force, such as a physician's hand, moves the limb, some muscles are stretched. Some resistance to such a movement is produced by the physical properties of the limb's tissues (muscles, tendons, skin, etc.). Additional resistance is caused by stretch reflexes. Sensory fibers detect lengthening of the muscles and cause motor neurons in the spinal cord to contract the same muscles. If the limb is truly relaxed, these responses are limited to short-latency reflexes (i.e., they appear 25-50 msec after the onset of the stretch), only involving circuits within the spinal cord. If the stretch is applied (Fig. 1A) when the muscle is contracting, additional reflexes contribute to resistance (Marsden et al. 1972; Shemmell et al. 2010). These are long-latency reflexes. They appear 50-100 msec after onset of the stretch and are seen as muscle activation that occurs after activity caused by spinal reflexes, but before any voluntary reaction to the passive movement (Fig. 1B).

A major contributor to rigidity in $\mathrm{PD}$ is an exaggeration of long-latency reflexes (Tatton and Lee 1975; Berardelli et al. 1983; Rothwell et al. 1983). These are larger than normal (Fig. 1C), and they can occur even if the stretch is applied when muscles are not contracting. This abnormality suggests that the BG contributes to the generation of long-latency reflexes. Does this physiologic basis of rigidity offer insights into rigidity as a possible motor control abnormality? The answer requires understanding what long-latency reflexes contribute to normal motor control. This question remains an area of intense investigation. Long-latency reflexes are generated in the cerebral hemispheres and thus can be influenced by a variety of information available to BG and other brain structures. A current theory of motor control, known as "optimal feedback control" (Todorov and Jordan 2002; Diedrichsen et al. 2010), suggests that for every movement, the motor system sets how easily the movement can be changed. If we reach for a wine glass that is sitting between two candles, for example, the hand's trajectory should be stable and precise. Should another person's arm 
P. Mazzoni et al.
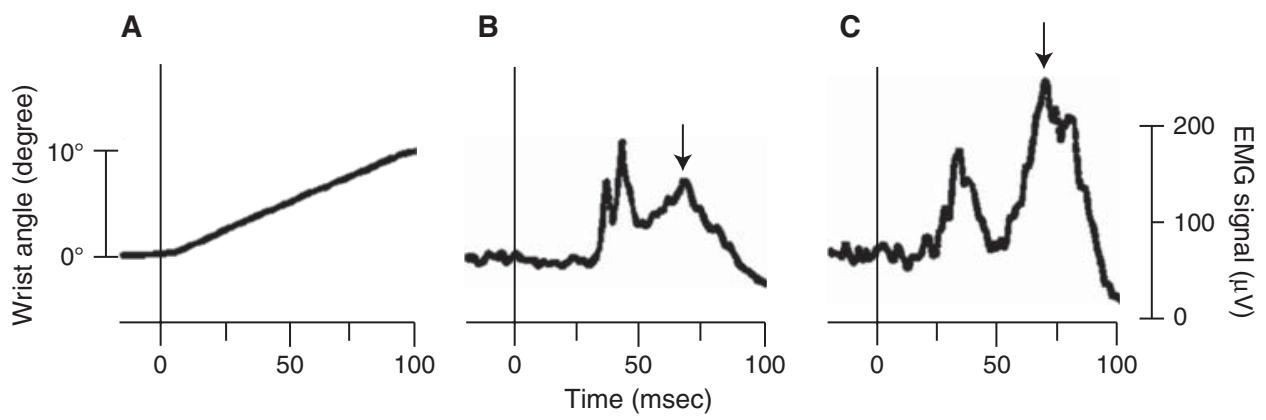

Figure 1. Long-latency reflexes are larger than normal in Parkinson's disease. The subject's hand is linked to a mechanical splint that suddenly displaces the hand so that the wrist is extended. This displacement, shown as a trace of wrist angle versus time $(A)$, causes a sudden stretch of muscles that flexes the wrist. This stretch elicits reflex muscle activity, shown in electromyographic recordings (EMG) from the flexor carpi radialis muscle for a healthy individual $(B)$ and a patient with PD $(C)$. Although the amplitudes of short-latency $(<50 \mathrm{msec})$ responses are similar for both subjects, the long-latency response ( $>50 \mathrm{msec})$ is exaggerated for the PD patient. (Adapted from Cody et al. 1986; reprinted, with permission, from Oxford University Press (C) 1986.)

accidentally bump into ours, we would want the arm to resist this perturbation. If, on the other hand, we are trying to catch a fruit fly that is weaving in many directions, the hand's trajectory should be flexible, at the expense of being less resistant to perturbations. Optimal feedback control theory posits that the brain sets "feedback gains," which determine how powerfully the hand resists changes in trajectory (Liu and Todorov 2007). Long-latency reflexes may play a role in setting feedback gains. They can be crafted specifically for a given limb, task, and conditions, but are "automatic" enough that they can occur earlier than voluntary responses.

Based on the hypothesis that muscle tone, mediated by long-latency reflexes, normally sets the amount of limb stability, it is possible to interpret parkinsonian rigidity at the level of motor control. If the basal ganglia contribute to the computation of normal tone required to maintain appropriate stability, then rigidity could be interpreted as a motor control dysfunction in which the limbs are programmed to be excessively stable.

\section{BRADYKINESIA, HYPOKINESIA, AND MOTOR CONTROL}

Bradykinesia, or movement slowing, is a prominent symptom of PD. When a patient with bradykinesia reaches for a cup on a table, the movement takes longer than for a person without PD. In addition, the initial movement is often too short. The hand briefly pauses short of the cup, and then additional small movements bring it to the cup. This undershooting is a manifestation of hypokinesia. Combined bradykinesia and hypokinesia can be elicited in the neurological examination. When a patient with PD is asked to tap his fingers "big and fast," the movements are both slower (fewer taps per second) and of smaller excursion than normal.

Bradykinesia and hypokinesia can be shown in the laboratory by recording an individual's fingertip position while he follows the instruction to tap the index finger and thumb "as big and as fast as possible" (Fig. 2). Compared with a healthy individual's finger taps (Fig. 2A), those of a $\mathrm{PD}$ patient have longer duration (bradykinesia) and reduced amplitude (hypokinesia) (Fig. 2B).

This slowing and reduction of movement amplitude were initially interpreted as a manifestation of weakness, which gave rise to the 19th century name for PD, "paralysis agitans," or shaking palsy (Parkinson 1817; Wilson 1925). The term "weakness," however, later assumed the more specific meaning of inability to produce a normal level of maximum force. PD does not cause weakness thus defined. This was clearly shown by Schwab et al. (1959), who asked a $\mathrm{PD}$ patient to repeatedly squeeze a rubber bulb 
A

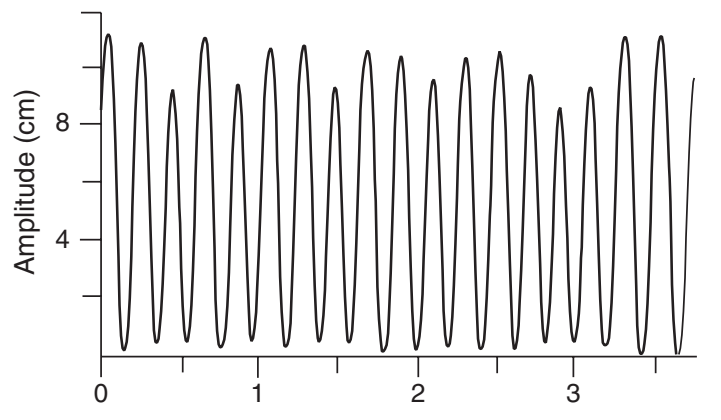

B

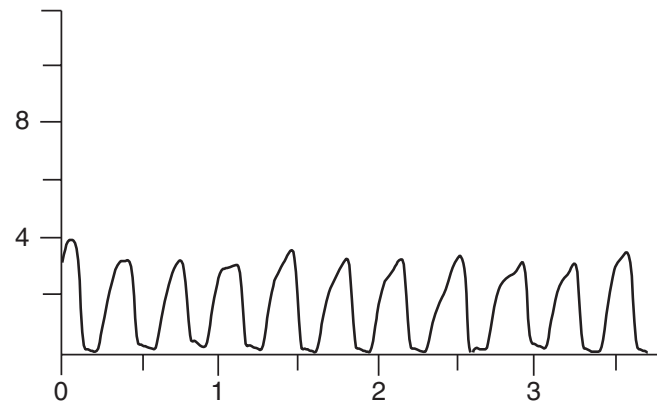

Figure 2. Bradykinesia and hypokinesia manifested in finger tapping. Individuals were asked to tap the index finger against the thumb "as big and as fast as possible." The traces show distance between the tips of the thumb and forefinger, recorded by motion capture camera, for a healthy individual $(A)$ and a patient with PD of similar age $(B)$. Finger taps for the PD patient were of smaller amplitude (hypokinesia) and lower frequency (bradykinesia) compared with those for the healthy individual. (Data courtesy of Drs. R. McGovern and F. DiBiasio.)

and recorded the pressure exerted. The pressure tracing showed a manifestation of hypokinesia known as "decrement," that is, a gradual decrease of the peak force with each repetition. However, if the examiner then reminded the patient to squeeze more forcefully, the pressure tracing immediately became larger. If the patient had been truly weak, then he should not have been able to increase the squeezing pressure without resting first. These findings were in contrast to those of a patient with myasthenia, a disorder of the neuromuscular junction that causes true weakness. The myasthenic patient was not able to increase the squeezing pressure after a verbal reminder, but instead needed to rest for a few seconds first. These findings showed that hypokinesia is not due to weakness.

Hypokinesia has been further studied through movement analysis in the laboratory. The typical approach is to instruct the subject to make specific goal-directed movements, such as moving the finger to a visual target as fast as possible, or moving a computer cursor with a joystick. Movements are recorded with high temporal resolution by a computer, so that a kinematic analysis can later be performed. This allows the extraction of movement parameters such as time of onset, speed, and amplitude. An important observation regarding hypokinesia and bradykinesia using this approach was made by studying joystick movements to guide a screen cursor to a visual target (Flowers 1975). The goal was to maintain the cursor on a visual target, which could randomly jump from one position to another. Healthy subjects moved the cursor in a single movement from its initial position to the target. Flowers noticed that the movements of PD patients were composed of an initial movement that fell short of the target, followed by additional corrective movements. The initial undershooting was a manifestation of hypokinesia, and it suggested a close relationship between bradykinesia and hypokinesia. If the initial movement is too short, then additional corrective movements are required, which causes the total movement duration to increase.

Flowers's finding fit within Woodworth's two-component model for the control of goaldirected movements (Woodworth 1899), according to which movements reflect a set initial plan followed by corrections, based on visual and proprioceptive feedback, as the hand's trajectory unfolds. In this scheme, Parkinson's causes the initial movement to be too short, that is, inadequately scaled to the distance to the target, which requires more time to be spent correcting for the initial undershooting. This insight, based on a motor control model of normal movements, not only supported the idea 
P. Mazzoni et al.

that bradykinesia and hypokinesia are related, but raised the question of what the primary deficit is. If movements are inadequately planned as too short and the extra time spent moving is due to the need for additional corrections, then the main deficit caused by PD would be inadequate scaling of the motor command, and a longer movement duration would be a consequence of this problem.

The scaling hypothesis states that $\mathrm{PD}$ patients can perform movements with a wide range of speeds and amplitudes, but inappropriately scale speed amplitude (i.e., the motor command) to the required goal distance (Berardelli et al. 1986). In a reaching task, a distant target might elicit a movement that is shorter and slower than normal. Such a movement would be perfectly adequate if the target were closer. When the target is placed closer, however, patients do not make the appropriate-size movement. Instead, they make a shorter, slower movement that is inadequate for the shorter target.

An important hypothesis, which emerged from subsequent studies of bradykinesia, is that the basal ganglia are responsible for "energizing" a movement, that is, for setting the correct size of motor commands so that muscles are adequately activated (Hallett and Khoshbin 1980). In support of this idea, it has been shown that PD patients produce normal muscle activation patterns, but the muscle activity is not adequately scaled to the required force (Berardelli et al. 1986; Turner and Desmurget 2010). These normal initial patterns are thus often followed by additional "corrective" muscle discharges in PD patients (Hallett and Khoshbin 1980). Flowers's data suggest that movements in PD have a normal initial organization (e.g., they have normal paths and velocity profiles) and are simply scaled down in speed and amplitude. The overall movement looks abnormal partly because it is composed of additional corrective movements that follow the initial underscaled movement.

Further support for this idea emerged from studies of arm reaching movements executed at different speeds (Mazzoni et al. 2007). Individuals made reaching movements to a visual target on a computer display, without seeing their hand during the movement. After each movement, the computer showed a marker indicating the hand's position at the end of the initial movement. In these conditions, patients with PD were able to make movements of the appropriate length, that is, without hypokinesia, and the movement's kinematic features (temporal profiles of velocity and acceleration) were nor$\mathrm{mal}$. These findings favor the idea that the motor system in PD patients can produce normal movements when specifically instructed to do so, but the disease causes a tendency for movements to be scaled down in speed and amplitude.

Why don't PD patients make movements of the required size if they are capable of doing so? This question was addressed in a study (Mazzoni et al. 2007) that asked the related question about normal motor control. Why don't healthy subjects move faster than they normally do? In other words, what determines normal movement speed? We hypothesized, in accordance with the computational theory of optimal feedback control, that the selection of movement speed (or the scaling of the motor command) is normally influenced by how much effort a movement requires. We showed that it is normal for speed to be selected based on required effort, a process that is driven by a form of motivation, which we called "motor motivation." The choice of this term was analogous to motivation for explicit choices such as pressing a lever for food (if you are a rat) or for accomplishing a long-term task (if you are a graduate student). We found that PD patients were less likely than healthy subjects to self-select fast movements, even though both groups performed with equal accuracy (Fig. 3). In other words, PD patients were not limited in the maximum speed they could achieve, but they selected lower speeds than normal. This selection was consistent with a higher sensitivity to the effort required by faster movements and thus suggested that PD causes a reduced level of motor motivation.

This result suggests a possible motor control framework for bradykinesia-hypokinesia, in which movement speed and amplitude are normally influenced by motor motivation, that is, by the implicit willingness to expend a certain amount of effort. In this framework, tonic 

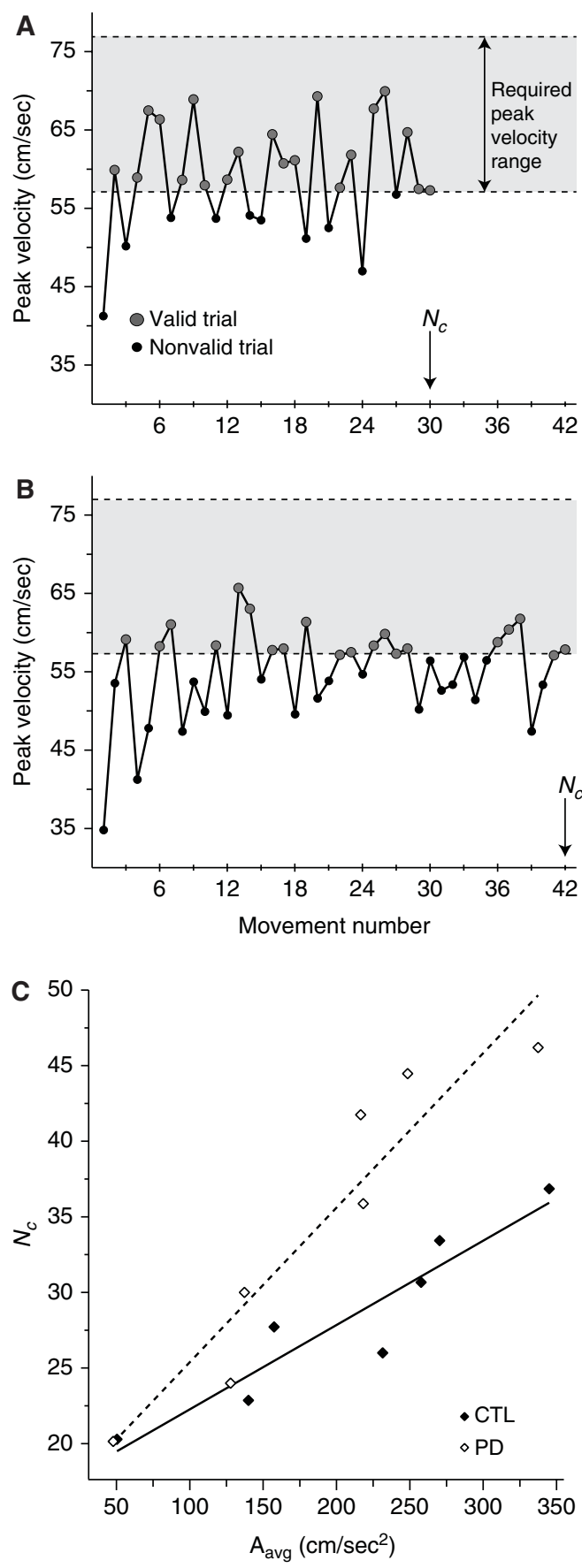

Figure 3. Parkinson's disease increases sensitivity to movement effort. Subjects made horizontal reaching movements to targets in a computer setup that provided feedback about movement speed. Movements that satisfied a given speed requirement were "valid" (gray circles), whereas all other movements dopamine in the striatum sets the level of motor motivation, and dopamine reduction in $\mathrm{PD}$ leads to bradykinesia and hypokinesia as manifestations of reduced scaling, or energizing, of movements.

It is important to note that we are not trying to indicate that $\mathrm{PD}$ reduces overall motivation or causes a form of laziness. Instead, patients perceive a normal task as requiring more effort than it should. Indeed, motor motivation may be separate from other types of motivation. Movement parameters, such as speed and amplitude, are selected automatically, without consciously thinking about them. Homework, a hobby, and running a marathon, on the other hand, are choices that we make with considerable explicit thought and are influenced by a more general behavioral motivation. Patients with PD often describe an increased sense of effort, without a loss of willingness to accomplish behavioral goals. We thus consider it advisable to treat motor motivation separately from other forms of behavioral motivation until their relationship is clarified.

Understanding bradykinesia-hypokinesia as a result of increased sensitivity to a movement's energy requirement makes it possible to understand certain aspects of PD that were previously difficult to explain. One of these is "kinesia paradoxica," the seemingly paradoxical ability of PD patients to move considerably faster than the maximum speed at which they could

Figure 3. (Continued) were "nonvalid" (black circles). Subjects made movements until 20 valid trials accumulated. The total number of trials needed to achieve this criterion, trials to criterion $\left(N_{\mathrm{C}}\right)$, was used as a measure of how much a subject was struggling in making movements at the required speed. For the same required speed, age-matched control subjects $(A)$ tended to make fewer nonvalid movements than $\mathrm{PD}$ patients $(B)$, and thus required fewer total trials to reach criterion. As the speed requirement increased, subjects from both groups needed more trials to reach criterion. Therefore, trials to criterion $\left(N_{\mathrm{C}}\right)$ depended on movement effort (quantified as average acceleration, $\left.\mathrm{A}_{\text {avg }}\right)(C)$. As indicated by the difference in slopes, PD patients showed higher sensitivity to movement effort than control subjects did. 
P. Mazzoni et al.

otherwise move (Souques 1921; Broussolle et al. 2010). The example typically given is that a patient with advanced PD, who is normally confined to a wheelchair, might be able to get up and run out of a theater if there is a fire. The situations described are characterized by a powerful urgent stimulus.

It has since been shown that this phenomenon is not peculiar to PD (Ballanger et al. 2006). Instead, it is normal for a powerful urgent stimulus to elicit a faster movement than a simple "go" signal does. Thus, the speed of a movement is influenced by two opposing forces: the urgency of a stimulus to move, and the motor system's sensitivity to the movement's required effort. Increasing the urgency leads both healthy subjects and PD patients to move faster than usual. Healthy subjects, who have a normal sensitivity to movement effort, move faster than normal; PD patients, who have greater sensitivity to effort, move at speeds that are closer to normal, that is, faster than their usual bradykinetic speeds.

\section{BRADYKINESIA AS ABNORMAL SPEED SELECTION}

In the past, a considerable amount of what we know about the motor system has been based on performance limits. Subjects in most studies are asked to move as fast as possible, or as accurately as possible, or in general to achieve best performance. Such studies allow us to understand certain disorders, such as stroke, in terms of how much ability these disorders remove from normal motor function. However, PD does not impair strength (Fahn 2003), and patients can usually move faster, or apply more force, when reminded to do so (Schwab et al. 1959). Instead, it is as if patients "select" abnormally slow movement speeds (Mazzoni et al. 2007). These considerations suggest that the core abnormality in bradykinesia should be described as a speed selection problem, and not as the loss of the ability to move at normal speeds. PD may cause patients to lower the speed that they consider normal.

To investigate this hypothesis in the context of normal motor control, we must identify the corresponding normal speed selection process. In other words, is there a "natural speed" for normal movements? Consider the act of reaching for a cup of water. We usually don't think about how fast we make this movement. The speed is selected automatically. However, we could deliberately reach more slowly than usual, and, if in a rush, we can deliberately reach faster. And yet, we easily notice whether someone is moving faster or slower than usual, which suggests that there is a typical speed for most movements, and that we are well attuned to it.

We recently looked for evidence for a speed preference by asking healthy subjects to reach for a visual target in one of two conditions, either at a "comfortable" speed, or at a speed instructed by a computer (B Shabbott and P Mazzoni, unpubl.). The remarkable finding was that subjects did show a preference for a particular speed. They returned to this speed even after they were shown that they could move faster or slower without a change in accuracy, and they moved at the same speed when they performed the task again the next day (Fig. 4). These findings support the existence of a natural speed in normal motor control, which is selected automatically and is resistant to the experience of other speeds. Bradykinesia could then be described as a reduction in the selected natural speed, due to increased sensitivity to movement effort.

Besides a speed preference for a particular reaching movement, we also found a relationship between a movement's amplitude and its natural speed. A specific natural speed seems to be matched to a given movement amplitude. Reaching for a target at a given distance thus elicits the selection of a particular natural speed, perhaps learned through practice or optimization of kinematic parameters. This yoking of speed and amplitude could explain the link between bradykinesia and hypokinesia observed in PD.

\section{AKINESIA AND MOTOR CONTROL}

The idea of scaling a motor command based on motor motivation combines speed and amplitude as linked aspects of movement. It thus offers an explanation for bradykinesia and hypokinesia 


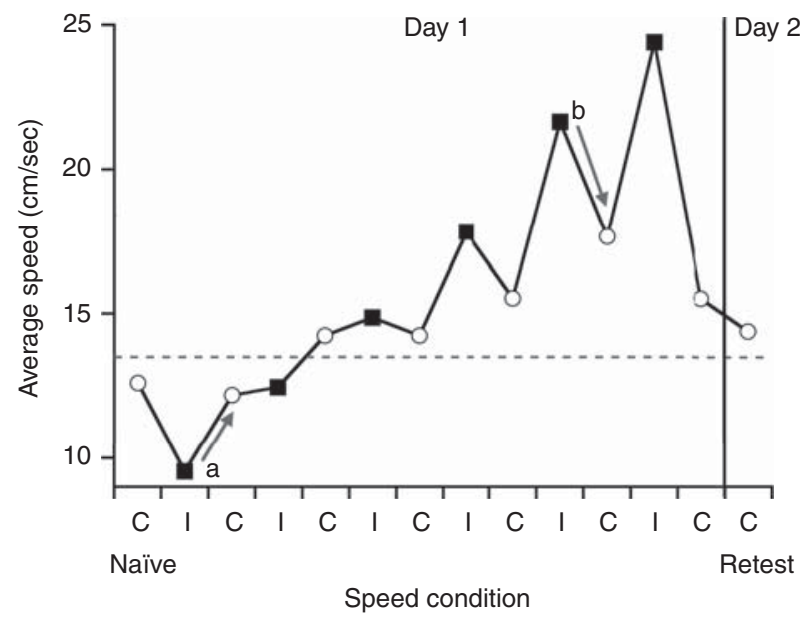

Figure 4. Healthy individuals prefer certain movement speeds. Subjects performed horizontal reaching movements to a target in a virtual reality environment equipped to record arm motion. Subjects completed alternating blocks of trials in which they reached with either their preferred "comfortable" speed (C, white circles) or with speeds imposed by a computer (I, black squares). Each subject's naïve speed preference (C naïve) was assessed before experiencing imposed speeds, and speed preference was also retested ( $\mathrm{C}$ retest) following a day of rest. The graph shows the mean average speed for a single subject. During comfortable speed blocks, the subject was reluctant to move at speeds that were either slower (see example, arrow a) or faster (see example, arrow b) than the naïve preference. The tendency to return to a certain preferred speed is shown by the arrows and occurs despite the fact that movements performed with all speeds resulted in similar accuracies. On the second day of testing, speed preference was similar to the naïve preference (the gray dotted line indicates the average of $\mathrm{C}$ naïve and $\mathrm{C}$ retest).

as two aspects of a single control problem. How does akinesia fit into this scheme? Although the term "akinesia" is often also used to describe reduced movement amplitude (which we have here referred to exclusively as "hypokinesia"), the other components of akinesia (paucity of spontaneous movements and delayed movement initiation) may or may not be related to hypokinesia or bradykinesia (Hallett 2003). In this discussion, we use "akinesia" specifically to refer to an abnormality in when a movement occurs. For repetitive automatic movements such as blinking and swallowing, this abnormality is manifest as a reduced frequency of these movements' occurrence. For voluntary movements, it is a delay in when the movement begins.

The motor motivation hypothesis (Mazzoni et al. 2007) is based on a modern theory of normal motor control, that is, optimal feedback control (Todorov and Jordan 2002), which predicts trajectory features of goal-directed movements. Similarly, it would be desirable to explain akinesia by resorting to theories that account for how movements are normally initiated and how the frequency of spontaneous movements, such as blinking and swallowing, is normally determined.

Unfortunately, motor control theories for movement initiation and spontaneous movement frequency are far less developed than those for the trajectories of voluntary movements. A motor control interpretation of akinesia, therefore, remains speculative. With respect to the frequency of cyclical automatic movements such as blinking and swallowing, very little is known at the control level. The benefits of these movements are clear, namely, clearing the eyes of lacrimal fluid and emptying the mouth of saliva, but these movements are not purely driven by the accumulation of these fluids. They have a central drive that is affected by basal ganglia dopamine levels, and their frequency can be affected by external factors (Ponder and Kennedy 1927; Lear et al. 1965; Karson 1983; Dodds 1989; Bentivoglio et al. 1997). However, a control-level account of these movements is lacking. 
P. Mazzoni et al.

Regarding delayed movement initiation, its possible relationship to normal motor control is suggested by a variety of findings. It is possible that this symptom is not related to bradykinesia, because the severity of initiation delay does not correlate with the severity of movement slowing (Evarts et al. 1981). Thus, delayed movement onset may be due to a separable physiological mechanism.

The most common method to establish speed of movement initiation is the reaction time (RT) paradigm. A "go" signal, such as an auditory tone or the appearance of a visual target, is given, and the subject is asked to make a movement as soon as possible; the time between the signal and movement onset is the RT. Typical values of RT for healthy older individuals are 300-500 msec for simple tasks, such as pressing a button as soon as possible after a light is turned on. RT is increased in PD, by an amount that ranges from $10 \%$ to $30 \%$ (Evarts et al. 1981). Measurements of RT in special conditions, such as requiring subjects to use the "go" signal to decide which button to press (choice RT paradigm), have shown that the RT increase caused by $\mathrm{PD}$ is specific to starting movement execution and does not seem to reflect a delay in other movement preparation processes (Jahanshahi et al. 1992).

The increase in RT is accompanied by slower buildup of neuronal activation over the motor cortex (Bereitschaftspotential, or "readiness" potential). The delay thus seems to reflect abnormally slow development of a motor command signal in the motor cortex. Once the motor command leaves the motor cortex, its transmission is normal (Dick et al. 1984). Therefore, PD affects the step between the preparation of a motor command and its manifestation in cortical motor areas. The basal ganglia receive information from multiple cortical areas and normally exert a net inhibitory action on the motor cortex (Alexander et al. 1986; Albin et al. 1989; Obeso et al. 2008). Just before a movement starts, this inhibitory action of the BG on motor cortex is transiently reduced. Transient disinhibition is blunted in PD, and this is part of the physiological basis of several PD symptoms. However, this physiological activity has not yet been integrated into a theory of how these signals determine normal movement onset. Until a better appreciation is gained of these normal processes, our understanding of akinesia will remain limited.

Akinesia could be related to bradykinesia if we hypothesize that movement initiation requires overcoming a certain "activation threshold" (Hallett 2003). If the motor cortex needs to be sufficiently "energized" for a movement to be of normal speed, then it may be necessary for this activation to cross a threshold value for a movement to start in the first place. This activation likely takes time to build up, as suggested by the time course of the readiness potential, an electrical signal recordable at the scalp that indicates activation of motor cortical areas before a movement starts (Deecke et al. 1976). In PD, the readiness potential builds up more slowly than normal (Shibasaki et al. 1978; Dick et al. 1989). The slower buildup of activation in PD patients could cause the activation threshold to be crossed later than normal, delaying movement initiation.

The reduction in frequency of spontaneous movement could also fit a threshold-crossing mechanism. Movements such as blinking and swallowing are performed automatically. When a movement becomes automatic, activity in motor cortical areas becomes reduced, which may reflect increased efficiency of neural coding for motor programs (Wu et al. 2004). Such activity would be expected to be further reduced in PD and thus may not cross the threshold required to generate a blink or swallowing. With increased time between these events, the stimulus to perform them might increase: buildup of lacrimal fluid over the eyes and accumulation of saliva in the mouth. These increased stimuli could generate sufficient motor drive to cross the activation threshold required for the movement to occur. Blinking and swallowing would thus still occur, but less frequently than normal.

\section{MOTOR SYMPTOMS OF PD AND NORMAL FUNCTION OF THE BG}

Behavior can be described at the level of actions or movements. A movement-level description emphasizes graded variables such as movement 
speed, amplitude, force, onset time, and duration. An action-level description emphasizes discrete chunks of behavior, such as what action was performed. The details of "how" a movement is performed (how fast, how long, etc.) are usually separable from descriptions of "what" the movement is (reaching for a cup or a fork at dinner, turning left or right in a maze). Remarkably successful descriptions of the role of BG in action selection have been developed (Redgrave et al. 1999). The research efforts that have led to these descriptions have encompassed multiple fields, including neuroanatomy, physiology, psychophysics, and computational theories. The result is a detailed tentative description of the BG as a "selection machine." Given a context and a behavioral goal, the BG may function to combine prior experience and probability of reward to guide the selection of the next action (Redgrave et al. 1999).

Despite the success of such theories in describing normal action selection, it has been difficult to reconcile these theories with the motor symptoms of PD. These symptoms mainly affect how a patient moves, not what action he or she performs. In general, movement-level descriptions of behavior have not included a role for selection processes. Kinematic parameters such as speed and amplitude are posited to be precisely computed based on spatial and mechanical aspects of movement, and not to be subject to a selection step. Consequently, computational theories of motor control have largely focused on obligatory relationships among kinematic variables, without entertaining a role for selection processes. However, selection of movement parameters is likely to play an important role in motor control.

The concept of natural speed is helpful in developing the concept of kinematic parameter selection. Daily experience with normal movements points to a selection process for speed. We are usually free to move faster or slower, which means that we must be choosing a particular speed. The nature of bradykinesia reinforces this idea. As described above, PD patients can move faster when reminded to (Schwab et al. 1959), and when they make faster movements, these are kinematically normal (Maz- zoni et al. 2007). Thus, bradykinesia may simply reflect a change in selection parameters, so that the speed that is selected as appropriate is lower than normal. This could occur, as suggested by our study, because of a change in sensitivity to the factors that normally influence speed selection (Mazzoni et al. 2007).

Conceptually, a view of normal speed and bradykinesia as results of selection processes opens the possibility of describing the function of BG as a selection machine for movement parameters, in analogy to its role in action selection. Such a conceptual framework would assign the common role of performing selections that guide behavior, both for "what" behavior is performed (action selection) and for "how" it is performed (motor control). A distinction between "what" and "how" has been proposed for the separate visual processing streams in temporal and parietal cortical areas (Goodale and Milner 1992). The basal ganglia may play an important role in integrating such perceptual information, and through its parallel anatomical circuits, may perform computationally analogous processes (selection based on context, motivation, reward, risk) to both aspects of behavioral control.

\section{CONCLUDING REMARKS}

The two PD symptoms discussed in this chapter-rigidity and the bradykinesia-hypokinesia-akinesia complex-illustrate the evolution of our understanding of these symptoms as motor control abnormalities. For these symptoms, the exciting possibility of linking movement abnormalities to theories of normal motor control is on the horizon. For other symptoms, such as tremor, we have an extensive understanding of the underlying physiological abnormalities (Hallett 2003), but it remains unclear how (or whether) they may be understood as motor control problems. It is important to recognize, and hopefully sufficiently clear from this article, however, that the process of understanding motor symptoms is not slave to understanding normal motor control first. On the contrary, in the case of bradykinesia, it was the remarkable aspects of the symptom that forced researchers to 


\section{P. Mazzoni et al.}

realize that it is possible to move slowly in the absence of weakness, and thus that speed might normally be governed by a selection process. Thus, even in the age of sophisticated computational theories of motor control, neurological symptoms continue to guide our understanding of normal brain function.

\section{REFERENCES}

Albin RL, Young AB, Penney JB. 1989. The functional anatomy of basal ganglia disorders. Trends Neurosci 12: $366-$ 375.

Alexander GE, DeLong MR, Strick PL. 1986. Parallel organization of functionally segregated circuits linking basal ganglia and cortex. Annu Rev Neurosci 9: 357-381.

Ballanger B, Thobois S, Baraduc P, Turner RS, Broussolle E, Desmurget M. 2006. "Paradoxical kinesis" is not a hallmark of Parkinson's disease but a general property of the motor system. Mov Disord 21: 1490-1495.

Bastian AJ. 2006. Learning to predict the future: The cerebellum adapts feedforward movement control. Curr Opin Neurobiol 16: 645-649.

Bastian AJ, Martin TA, Keating JG, Thach WT. 1996. Cerebellar ataxia: Abnormal control of interaction torques across multiple joints. J Neurophysiol 76: 492-509.

Benecke R, Rothwell JC, Dick JP, Day BL, Marsden CD. 1987. Disturbance of sequential movements in patients with Parkinson's disease. Brain 110: 361-379.

Bentivoglio A, Bressman S, Cassetta E, Carretta D, Tonali P, Albanese A. 1997. Analysis of blink rate patterns in normal subjects. Mov Disord 12: 1028-1034.

Berardelli A, Sabra AF, Hallett M. 1983. Physiological mechanisms of rigidity in Parkinson's disease. J Neurol Neurosurg Psychiatry 46: 45-53.

Berardelli A, Dick JP, Rothwell JC, Day BL, Marsden CD. 1986. Scaling of the size of the first agonist EMG burst during rapid wrist movements in patients with Parkinson's disease. J Neurol Neurosurg Psychiatry 49: 1273-1279.

Bernstein NA. 1967. The coordination and regulation of movements. Pergamon, New York.

Broussolle E, Loiraud C, Thobois S. 2010. Achille Alexandre Souques (1860-1944). J Neurol 257: 1047-1048.

Brunner P, Bianchi L, Guger C, Cincotti F, Schalk G. 2011. Current trends in hardware and software for braincomputer interfaces (BCIs). J Neural Eng 8: 025001.

Cody FW, MacDermott N, Matthews PB, Richardson HC. 1986. Observations on the genesis of the stretch reflex in Parkinson's disease. Brain 109: 229-249.

Deecke Ld, Grözinger B, Kornhuber HH. 1976. Voluntary finger movement in man: Cerebral potentials and theory. Biol Cybern 23: 99-119.

Dick JP, Cowan JM, Day BL, Berardelli A, Kachi T, Rothwell JC, Marsden CD. 1984. The corticomotoneurone connection is normal in Parkinson's disease. Nature 310: 407-409.

Dick JPR, Rothwell JC, Day BL, Cantello R, Buruma O, Gioux M, Benecke R, Berardelli A, Thompson PD, Mars- den CD. 1989. The Bereitschaftspotential is abnormal in Parkinson's disease. Brain 112: 233-244.

Diedrichsen J, Shadmehr R, Ivry RB. 2010. The coordination of movement: Optimal feedback control and beyond. Trends Cogn Sci 14: 31-39.

Dodds W. 1989. The physiology of swallowing. Dysphagia 3: $171-178$

Elftman H. 1939. The function of the arms in walking. Johns Hopkins Press, Baltimore.

Evarts EV, Teravainen H, Calne DB. 1981. Reaction time in Parkinson's disease. Brain 104: 167-186.

Fahn S. 2003. Description of Parkinson's disease as a clinical syndrome. Ann NY Acad Sci 991: 1-14.

Ferrier D. 1876. The functions of the brain. G.P. Putnam's Sons, New York.

Flowers K. 1975. Ballistic and corrective movements on an aiming task. Intention tremor and parkinsonian movement disorders compared. Neurol 25: 413-421.

Goodale MA, Milner AD. 1992. Separate visual pathways for perception and action. Trends Neurosci 15: 20-25.

Hallett M. 1990. Clinical neurophysiology of akinesia. Rev Neurol (Paris) 146: 585-590.

Hallet M. 2003. Parkinson revisited: Pathophysiology of motor signs. Adv Neurol 91: 19-28.

Hallett M, Khoshbin S. 1980. A physiological mechanism of bradykinesia. Brain 103: 301-314.

Holmes G. 2007. The Croonian Lectures on the clinical symptoms of cerebellar disease and their interpretation. Lecture II. 1922. Cerebellum 6: 148-153.

Jahanshahi M, Brown RG, Marsden CD. 1992. Simple and choice reaction time and the use of advance information for motor preparation in Parkinson's disease. Brain 115: $539-564$.

Karson CN. 1983. Spontaneous eye-blink rates and dopaminergic systems. Brain 106: 643-653.

Kawato M, Furukawa K, Suzuki R. 1987. A hierarchical neural-network model for control and learning of voluntary movement. Biol Cybern 57: 169-185.

Lear CS, Flanagan JB, Moorrees CF. 1965. The frequency of deglutition in man. Arch Oral Biol 10: 83-100.

Liu SC, Delbruck T. 2010. Neuromorphic sensory systems. Curr Opin Neurobiol 20: 288-295.

Liu D, Todorov E. 2007. Evidence for the flexible sensorimotor strategies predicted by optimal feedback control. J Neurosci 27: 9354-9368.

Marr D. 1982. Vision. Freeman, New York.

Marsden CD. 1989. Slowness of movement in Parkinson's disease. Mov Disord 4: S26-S37.

Marsden CD, Merton PA, Morton HB. 1972. Servo action in human voluntary movement. Nature 238: 140-143.

Mazzoni P, Hristova A, Krakauer JW. 2007. Why don't we move faster? Parkinson's disease, movement vigor, and implicit motivation. J Neurosci 27: 7105-7116.

Miall RC, Wolpert DM. 1996. Forward models for physiologic motor control. Neural Networks 9: 1265-1279.

Miall RC, Weir DJ, Wolpert DM, Stein JF. 1993. Is the cerebellum a Smith Predictor? J Mot Behav 25: 203-216.

Obeso JA, Marin C, Rodriguez-Oroz C, Blesa J, BenitezTemino B, Mena-Segovia J, Rodriguez M, Olanow CW. 
2008. The basal ganglia in Parkinson's disease: Current concepts and unexplained observations. Ann Neurol 64: S30-S46.

Parkinson J. 1817. An essay on the shaking palsy. Wittingham \& Rowland, London.

Ponder E, Kennedy WP. 1927. On the act of blinking. Exp Physiol 18: 89-110.

Redgrave P, Prescott TJ, Gurney K. 1999. The basal ganglia: A vertebrate solution to the selection problem? Neuroscience 89: 1009-1023.

Rothwell JC, Obeso JA, Traub MM, Marsden CD. 1983. The behaviour of the long-latency stretch reflex in patients with Parkinson's disease. J Neurol Neurosurg Psychiatry 46: 35-44.

Schwab RS, Chafetz ME, Walker E. 1954. Control of two simultaneous motor acts in normals and in Parkinsonism. AMA Arch Neurol Psychiatry 72: 591-598.

Schwab RS, England AC, Peterson E. 1959. Akinesia in Parkinson's disease. Neurol 9: 65-72.

Scott S. 2003. The role of primary motor cortex in goaldirected movements: Insights from neurophysiological studies on non-human primates. Curr Opin Neurobiol 13: $671-677$.

Scott SH. 2008. Inconvenient truths about neural processing in primary motor cortex. J Physiol 586: 1217-1224.

Shadmehr R, Wise SP. 2005. Computational neurobiology of reaching and pointing: A foundation for motor learning. MIT Press, Cambridge, MA.

Shemmell J, Krutky M, Perreault E. 2010. Stretch sensitive reflexes as an adaptive mechanism for maintaining limb stability. Clin Neurophysiol 121: 1680-1689.
Shibasaki H, Shima F, Kuroiwa Y. 1978. Clinical studies of the movement-related cortical potential (MP) and the relationship between the dentatorubrothalamic pathway and readiness potential (RP). J Neurol 219: 15-25.

Souques A-A. 1921. Rapport sur les syndromes parkinsoniens. Rev Neurol 37: 181.

Tatton WG, Lee RG. 1975. Evidence for abnormal long-loop reflexes in rigid Parkinsonian patients. Brain Res 100: 671-676.

Thach WT. 1978. Correlation of neural discharge with pattern and force of muscular activity, joint position, and direction of intended next movement in motor cortex and cerebellum. J Neurophysiol 41: 654-676.

Todorov E, Jordan MI. 2002. Optimal feedback control as a theory of motor coordination. Nat Neurosci 5: 12261235.

Turner R, Desmurget M. 2010. Basal ganglia contributions to motor control: A vigorous tutor. Curr Opin Neurobiol 20: 704-716.

Wilson SAK. 1925. The Croonian Lectures on some disorders of motility and of muscle tone with special reference to the corpus striatum. Lancet 2: 1-10, 53-62.

Winter DA. 2009. Biomechanics and motor control of human movement. Wiley, New York.

Wolpert DM, Ghahramani Z, Jordan MI. 1995. An internal model for sensorimotor integration. Science 269: 1880 1882.

Woodworth RS. 1899. The accuracy of voluntary movement. Psychol Rev 3: 1-119.

Wu T, Kansaku K, Hallett M. 2004. How self-initiated memorized movements become automatic: A functional MRI study. J Neurophysiol 91: 1690-1698. 


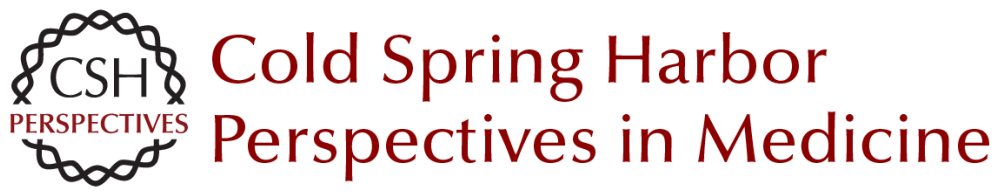

\section{Motor Control Abnormalities in Parkinson's Disease}

Pietro Mazzoni, Britne Shabbott and Juan Camilo Cortés

Cold Spring Harb Perspect Med 2012; doi: 10.1101/cshperspect.a009282 originally published online March 27, 2012

\section{Subject Collection Parkinson's Disease}

Functional Neuroanatomy of the Basal Ganglia José L. Lanciego, Natasha Luquin and José A. Obeso

Animal Models of Parkinson's Disease: Vertebrate Genetics Yunjong Lee, Valina L. Dawson and Ted M. Dawson

Innate Inflammation in Parkinson's Disease V. Hugh Perry

Parkinson's Disease and Parkinsonism:

Neuropathology Dennis W. Dickson

Physiological Phenotype and Vulnerability in Parkinson's Disease

$D$. James Surmeier, Jaime N. Guzman, Javier Sanchez, et al.

Clinical Approach to Parkinson's Disease:

Features, Diagnosis, and Principles of Management João Massano and Kailash P. Bhatia

The Role of Autophagy in Parkinson's Disease Melinda A. Lynch-Day, Kai Mao, Ke Wang, et al.

Disruption of Protein Quality Control in Parkinson's Disease Casey Cook, Caroline Stetler and Leonard Petrucelli
Drosophila as a Model to Study Mitochondrial Dysfunction in Parkinson's Disease Ming Guo

Parkinsonism Due to Mutations in PINK1, Parkin, and DJ-1 and Oxidative Stress and Mitochondrial Pathways Mark R. Cookson

Programmed Cell Death in Parkinson's Disease Katerina Venderova and David S. Park

Genomics and Bioinformatics of Parkinson's

Disease Sonja W. Scholz, Tim Mhyre, Habtom Ressom, et al.

Motor Control Abnormalities in Parkinson's

Disease Pietro Mazzoni, Britne Shabbott and Juan Camilo Cortés

Parkinson's Disease: Gene Therapies Philippe G. Coune, Bernard L. Schneider and Patrick Aebischer

Functional Neuroimaging in Parkinson's Disease Martin Niethammer, Andrew Feigin and David Eidelberg

Leucine-Rich Repeat Kinase 2 for Beginners: Six Key Questions Lauren R. Kett and William T. Dauer

For additional articles in this collection, see http://perspectivesinmedicine.cshlp.org/cgi/collection/ 